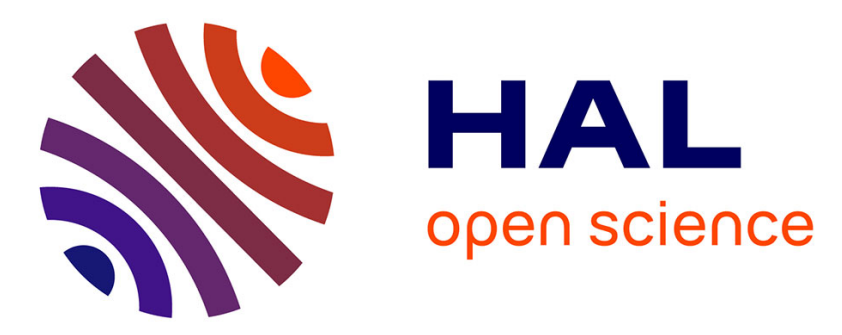

\title{
Éléments pour une approche écologique du travail des interprètes auprès de migrants : penser cette pratique dans ce qu'elle a de risqué
}

\author{
Anne-Sophie Haeringer
}

\section{To cite this version:}

Anne-Sophie Haeringer. Éléments pour une approche écologique du travail des interprètes auprès de migrants : penser cette pratique dans ce qu'elle a de risqué. Rhizome , 2020, Nº75-76 (1), pp.120. 10.3917/rhiz.075.0120 . halshs-02918904

\section{HAL Id: halshs-02918904 \\ https://shs.hal.science/halshs-02918904}

Submitted on 5 Oct 2020

HAL is a multi-disciplinary open access archive for the deposit and dissemination of scientific research documents, whether they are published or not. The documents may come from teaching and research institutions in France or abroad, or from public or private research centers.
L'archive ouverte pluridisciplinaire HAL, est destinée au dépôt et à la diffusion de documents scientifiques de niveau recherche, publiés ou non, émanant des établissements d'enseignement et de recherche français ou étrangers, des laboratoires publics ou privés. 


\title{
ÉLÉMENTS POUR UNE APPROCHE ÉCOLOGIQUE DU TRAVAIL DES INTERPRĖTES AUPRĖS DE MIGRANTS : PENSER CETTE PRATIQUE DANS CE QU'ELLE A DE RISQUÉ
}

\author{
Anne-Sophie Haeringer \\ Orspere-Samdarra | « Rhizome»
}

2020/1 N 75-76 | pages 120 à 131

ISSN 1622-2032

Article disponible en ligne à l'adresse :

https://www.cairn.info/revue-rhizome-2020-1-page-120.htm

Distribution électronique Cairn.info pour Orspere-Samdarra.

(C) Orspere-Samdarra. Tous droits réservés pour tous pays.

La reproduction ou représentation de cet article, notamment par photocopie, n'est autorisée que dans les limites des conditions générales d'utilisation du site ou, le cas échéant, des conditions générales de la licence souscrite par votre établissement. Toute autre reproduction ou représentation, en tout ou partie, sous quelque forme et de quelque manière que ce soit, est interdite sauf accord préalable et écrit de l'éditeur, en dehors des cas prévus par la législation en vigueur en France. Il est précisé que son stockage dans une base de données est également interdit. 


\section{Anne-Sophie Haeringer}

\section{Sociologue, enseignante-chercheure}

Université Lyon II

PoCo-CMW (UMR 5283, CNRS)
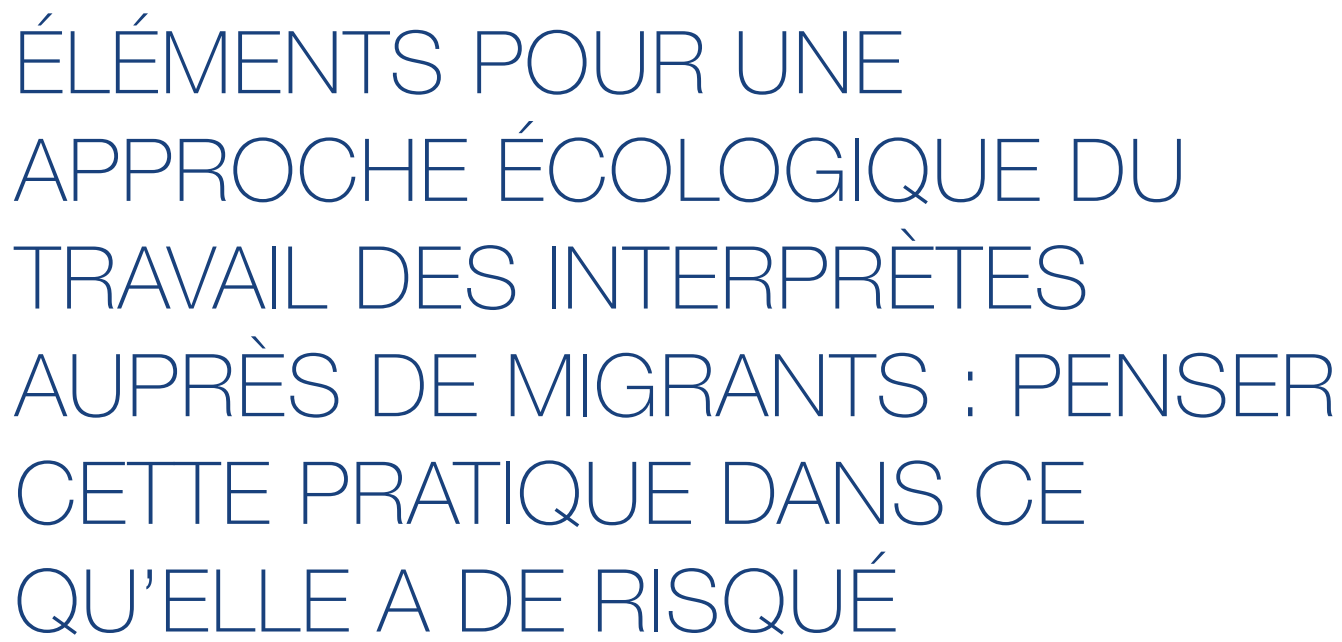

1 Lors de ces formations organisées par l'OrspereSamdarra, nous donnons accès à une retraduction assez littérale des propos tenus dans l'autre langue. Tout un chacun peut ainsi comparer les différents énoncés entre eux, quand bien même il ne parle pas la langue en question.
"C'est mal traduit! ", telle est l'une des premières exclamations que la projection d'un extrait vidéo d'une consultation médicale réunissant une psychologue, une interprète et un patient angolais a suscitées chez une interprète lusophone qui participait à une formation portant sur "l'interprétariat en santé ».

"Ça fait peur! ", telle est la réaction d'un médecin qui découvre, lors d'une de ces formations, l'écart qui existe entre ce que la psychologue dit en français et ce qui est traduit en portugais au patient¹.

Pourtant, ces écarts ont été repérés depuis longtemps par tous ceux qui s'intéressent à la traduction. Ils constituent le cœur même de cet art et ont, à ce titre, fait l'objet de nombreux déploiements théoriques, que l'on songe, entre autres, aux travaux de Barbara Cassin sur la "barbarie " des "intraduisibles " qui visent à "compliquer l'universel " et à résister à la réduction du pluralisme des langues (2016). Est-ce à dire qu'il y aurait écart et écart? Que les interprètes et les soignants que je rencontre adhéreraient au contraire à 
une conception (à la fois très pauvre et politiquement problématique) de la traduction comme identification et mêmeté, qui se doit de surpasser les différences et de produire le même discours dans une autre langue ? Y aurait-il un traitement différencié de la traduction suivant que l'on se situe dans un cadre poétique et littéraire, ouvert à la créativité, voire à la part d'auteur du traducteur, ou dans un cadre social ou médical attaché au sens littéral, où il s'agirait seulement d'interpréter?

Cette partition entre les scènes est un peu suspecte. II suffit de considérer la place que l'ethnopsychiatrie réserve à ses interprètes. Au cours des consultations en ethnopsychiatrie, les difficultés de traduction et les malentendus sont discutés collectivement et publiquement par les thérapeutes, les interprètes - qui sont à ce point intégrés au dispositif thérapeutique qu'ils sont rebaptisés en "traducteurs ou médiateurs ethnocliniciens " - et les patients, qui sont eux aussi transformés du fait qu'une expertise leur est ainsi reconnue, en ce que l'élucidation du choix des mots est tout autant leur affaire (de Pury Toumi, 2005). Qualifier ces interprètes de "médiateurs ", c'est faire valoir qu'ils ne sont pas de simples intermédiaires, mais qu'ils transforment la situation et les êtres en présence (Hennion, 2015).

Force est de constater toutefois que, en regard des consultations auxquelles je me suis intéressée au sein de l'équipe mobile psychiatrie précarité (EMPP)

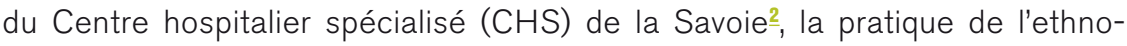
psychiatrie présente bien des aspects exceptionnels. Alors que cette dernière concerne plutôt des personnes qui viennent à ces consultations après avoir épuisé d'autres dispositifs sociaux ou médicaux plus classiques, l'EMPP est un dispositif de droit commun. Ses consultations s'adressent au tout venant

$\underline{2}$ Cette enquête s'inscrit dans le cadre du projet " Réfugiés, migrants et leurs langues face aux services de santé » (Remilas) financé par I'ANR (16-Fasi-0003).

3 Extrait du site internet de l'ADDCAES (Association Départementale pour le Développement et la Coordination des Actions auprès des Étrangers de la Savoie) : https://www. addcaes.org/qui-sommesnous/ précaire. Les interprètes ne sont pas liés à l'EMPP, mais à une association qui développe des "actions afin de contribuer à l'intégration des immigrés et de leurs familles, à l'accès aux droits et à l'égalité des chances ${ }^{3}$ ». Ils n'ont pas été formés à une clinique particulière. Enfin, lorsqu'ils sont sollicités, c'est toujours pour intervenir auprès de migrants allophones, dans des situations souvent marquées par l'urgence.

L'inquiétude qui saisit certains soignants qui regardent les vidéos de ces consultations tient peut-être d'abord à ce qui leur apparaît fort justement comme une absence de maîtrise. Alors que le dispositif ethnopsychiatrique internalise les problèmes de traduction, il n'y a rien de tel dans les consultations que j'ai observées. 
Quant aux critiques formulées par certains interprètes lors du visionnage de ces vidéos, elles témoignent pour leur part sans doute surtout de la conception assez fonctionnelle et instrumentale de la communication et de la traduction qui prévaut dans les situations que j'observe. C'est là ce que pointe aussi l'expression utilisée par une interprète filmée que j'ai rencontrée : "Je suis une petite cassette. " Cette manière de parler de son travail ne fait toutefois pas nécessairement signe vers une réduction des écarts entre les énoncés produits dans une langue et ceux traduits dans une autre, par une résolution mécanique qui rendrait enfin possible la traduction littérale. Elle témoigne aussi bien d'abord de la modestie du travail de ces interprètes et des conditions de félicité d'un travail qui consiste, pour ceux qui l'exercent, à tout faire pour s'effacer. L'immédiateté n'est pas la disparition des médiateurs. Elle demande au contraire à être pensée comme un effet de leur alignement heureux (Hennion, 2015). II suffit qu'un médiateur soit désaxé, par exemple qu'un interprète achoppe sur la traduction d'un terme, tâtonne ou balbutie un peu trop pour que ce soient d'abord ses bégaiements et son travail pour les négocier qui soient rendus visibles plutôt que l'énoncé traduit.

Dans cet article, je ne chercherai donc surtout pas à rassurer ni les uns ni les autres, pas plus que je ne lèverai les inquiétudes que génère l'existence de tels écarts. Plutôt que de mettre en évidence ce qui constituerait l'art spécifique de ces interprètes et de souligner leur maîtrise ou leur virtuosité, par exemple en célébrant leur sens du bricolage, j'aimerais décrire leur activité à même sa vulnérabilité, c'està-dire en tant qu'elle se situe toujours sur une arête. Ce faisant, j'entends penser conjointement l'art qui est le leur et la possibilité de sa faillite. Je montrerai ainsi comment certaines solutions trouvées pour négocier les difficultés rencontrées risquent toujours de créer de nouveaux problèmes. Dit autrement, je montrerai que ces situations éminemment complexes ne connaissent pas nécessairement d'issue heureuse, a fortiori durablement, mais que l'inconfort qu'elles suscitent ne doit pas être considéré comme un problème. Ma contribution en tant que chercheure à cet univers de pratiques consiste finalement à penser ce trouble, c'est-à-dire à explorer des manières de l'habiter"․․

Pour cela, je m'appuierai sur un dispositif méthodologique que j'ai mis en place: je suis retournée voir les interprètes et soignants filmés dans le cadre du projet "Réfugiés, migrants et leurs langues face aux services de santé " (Remilas) et leur ai fait commenter les " traces de leur activité »(Cahour et Licoppe, 2010). Dans l'espace qui m'est imparti ici, je mobiliserai plus spécifiquement un extrait d'une consultation filmée et les discussions que j'ai eues avec l'interprète en le visionnant. 


\section{Du caractère éminemment composite de l'activité de l'interprète}

La consultation réunit une psychiatre, un couple de Serbes venu avec leur enfant et une interprète d'origine macédonienne qui parle pas moins de sept langues différentes, dont le serbe. La consultation dure une trentaine de minutes. Si l'interprète et la psychiatre se connaissent déjà et ont des habitudes de travail, c'est la troisième fois qu'elles rencontrent cette famille.

Alors que la consultation a démarré depuis trois ou quatre minutes, et avant d'aborder plus spécifiquement les questions de santé, la psychiatre fait le tour des aspects " matériels » de leur situation (hébergement et " histoires de papiers ").

Le couple répond, l'interprète traduit. Elle dit qu'ils sont toujours hébergés en centre d'accueil pour demandeurs d'asile (Cada), qu'ils attendent la convocation de l'Office français de protection des réfugiés et apatrides (Ofpra).

La psychiatre interpelle alors l'enfant: "Et toi, tu vas à l'école?»

L'enfant ne répond pas, peut-être n'en a-t-il pas le temps, car la femme intervient aussitôt: "Je voulais vous demander encore quelque chose, est-ce qu'elle peut me donner un papier à donner personnellement à l'Ofpra qu'ils ne nous demandent pas trop, qu'ils ne nous interrogent pas trop, vous savez comme c'est quand vous allez là-bas [silence]. Vous allez sûrement écrire ${ }^{5} . . . "$

L'interprète ne traduit d'abord pas la demande. Elle interroge le couple en retour : "Elle [la psychiatre] l'a fait pour le dossier, non? les papiers?"

5 Pour ne pas alourdir la lecture, je ne produis pas ici la transcription serbe des propos tenus par le couple ou l'interprète mais directement la (re) traduction française proposée par Amandine Potelle, traductrice recrutée dans le cadre du projet Remilas.
La femme répond: "[inaudible] Elle [sans doute l'intervenante sociale du Cada] a reçu un, je veux dire, dossier... Nous avons reçu au Cada un dossier, mais moi je ne comprends pas ce qui est écrit."

L'interprète s'adresse à la psychiatre : "Elle veut savoir si elle peut obtenir un papier de votre part pour le jour qu'elle va se présenter làbas, de donner, je pense que vous avez, je..." 
La psychiatre interrompt l'interprète : "Alors c'est déjà fait. "

L'interprète : "C'est déjà fait, voilà, c'est pour ça que je me suis permis... "

La psychiatre : "Oui, oui. »

Sans attendre la traduction de l'interprète, l'homme intervient: "Excusez-moi, je suis très tête en l'air, je ne me rappelle pas de tout, j'ai des absences, vous comprenez. Depuis ce..."

Sa femme l'interrompt: "II arrive souvent qu'il se perde pendant la conversation et qu'il ne sache plus de quoi on parle et même parfois..." "

L'interprète interroge avant de traduire : "Est-ce que c'est nouveau ça ou ça arrivait avant? »

La femme répond: "Ça arrivait avant, mais maintenant, c'est vraiment souvent. »

L'interprète revient vers la psychiatre : "Oui, elle veut dire que maintenant, ce dernier temps, comme on est en train de parler, il se perd, il sait pas où elle est le sujet."

La psychiatre acquiesce pendant que l'interprète parle : "Oui, d'accord. ". Puis elle ajoute: "Alors on va, d'accord, on va voir ça.»

Sans attendre que la psychiatre ait terminé, la femme intervient à nouveau : "Pas toujours, mais par moments, vous savez, il arrive que sa tête se bloque. "

L'interprète traduit : "Ça dure pas longtemps, mais voilà. "

La psychiatre série les problèmes : "D'accord. Alors y a deux choses différentes."

Profitant d'un bref silence, l'interprète traduit : " Deux choses différentes, elle dit." 
La psychiatre poursuit : "Pour le dossier de l'Ofpra, les papiers à fournir, [...] donc moi j'ai déjà fait un certificat le [date] que j'ai transmis à [l'intervenante sociale du Cada]. »

La femme acquiesce. Peut-être a-t-elle reconnu le nom.

La psychiatre: "Pour le dossier de l'Ofpra. C'est du côté des papiers on va dire hein. Donc c'est..."

L'interprète traduit : "Elle dit pour les papiers, pas pour ce que tu as demandé, elle nous dira après. Pour les papiers que tu as demandés, elle dit qu'elle a tout envoyé. Moi j'ai fait une attestation [potvrda]. »

Elle ajoute en français " certificat».

La psychiatre poursuit : "Voilà où j'ai insisté, je résume simplement, sur la gravité de l'état de santé de votre mari. "

À ce moment de la consultation, la femme et son mari interviennent l'un après l'autre, sans répondre à une question posée par l'interprète ou la psychiatre comme c'est le cas le reste du temps. Lorsque la femme formule sa demande, la psychiatre vient de poser une question à leur enfant. Lorsque le mari évoque ses problèmes de santé, l'interprète n'a pas encore eu le temps de traduire l'échange qu'elle vient d'avoir avec la psychiatre à propos de la demande de sa femme.

S'ils prennent tous deux la parole successivement, sans attendre leur tour, c'est pour demander que la psychiatre indique dans son certificat que le mari présente des troubles cognitifs susceptibles d'entraver les réponses qu'il devra donner à l'officier de l'Ofpra. Ils adressent leur requête à deux voix : quand bien même l'intervention du mari n'est pas explicitement articulée à celle de sa femme, elle s'apparente à une justification de la demande formulée par celle-ci.

Toutefois, cette demande n'est pas vraiment entendue ni par l'interprète ni par la psychiatre. La question posée par la femme embarrasse en effet beaucoup l'interprète. Lorsque nous visionnons ensemble cet extrait, elle s'exclame d'emblée : 
"Elle veut un papier pour que le docteur Gekiere [la psychiatre] envoie là-bas [elle souffle]. Ils sont... Certaines familles... C'est bidon! Pour pas qu'ils [leur] posent trop de questions. Non, mais! [Son ton est offusqué]. »

Quand je la questionne plus avant sur ce qui lui pose problème, elle fait état de ce qu'il " est gênant " pour elle de relayer la demande de la femme à la psychiatre. Sa réticence se situe sur un plan moral, non pas parce qu'elle soupçonnerait cette femme de chercher à resquiller, mais parce qu'en traduisant sa demande, elle manifesterait d'abord son ignorance des procédures administratives et qu'elle risquerait en outre de mettre la psychiatre dans l'embarras. Car, à sa connaissance, le "papier pour pas [qu'on me] pose des questions" n'a aucune espèce d'existence administrative. Seul compte le "certificat médical ". L'interprète souligne d'ailleurs en entretien qu'elle prend bien soin de traduire "certificat " par le terme exact : potvdra.

De son côté, la psychiatre ne laisse pas tout à fait le temps à l'interprète d'aller au bout de sa traduction. En considérant pour sa part que "papier» est une forme vulgarisée ou approximative du terme " certificat " et en ne s'y arrêtant pas davantage, elle fait tout à la fois la preuve de son efficacité et de son savoir en matière de certification ("C'est déjà fait!»). Ce faisant, elle ne peut que conforter l'interprète dans l'impression que ses différentes anticipations sont pertinentes : efficacité et savoir de la psychiatre, inconvenance de la demande de la femme, inexistence administrative de ce fameux "papier", le terme ne faisant tout simplement pas partie du vocabulaire de la psychiatre.

Si ni l'interprète ni la psychiatre n'entendent vraiment la requête de ce couple, c'est aussi eu égard au fait qu'il va de soi pour elles que, en faisant état de ses " absences", le mari est en train de changer de sujet. Leur difficulté à voir que les propos du mari s'articulent à ceux de sa femme tient sans doute pour une part à ce que, en parlant sans attendre leur tour, l'homme et la femme contribuent quelque peu à désynchroniser l'échange, et pour une autre à ce qu'au tout début de la consultation, le mari a déjà été repris sur ce point par la psychiatre. Alors que celle-ci a amorcé sa consultation comme elle le fait toujours, en demandant "Comment ça va?", le mari a embrayé sur ses peurs. La psychiatre a aussitôt proposé d'organiser la consultation dans un ordre sensiblement différent, en commençant par aborder "les histoires de papiers " offrant du même coup la possibilité à la femme de la solliciter juste après pour obtenir un "papier [papir] [pour] qu'ils ne nous interrogent pas trop »-, avant 
que de parler de ces "pensées " qui hantent le mari et qui l'ont amené à se rendre dans cette consultation. Aussi bien donc, cette évocation par le mari de ses problèmes de santé ne peut-elle être entendue que comme une répétition, voire comme une forme de ressassement, qui le rend incapable de saisir l'ordre dont procède la consultation.

En insistant un peu maladroitement auprès de l'interprète sur ces restes qu'elle n'aurait pas vraiment traduits, j'oblige celle-ci à déplier certains autres éléments sur lesquels elle fait porter son attention et que je n'arrivais pas vraiment à voir. À défaut de tous les répertorier ici, je mentionnerai seulement le souci qu'elle a de permettre à la psychiatre d'accéder au bref aparté qu'elle a eu avec la femme ou encore de susciter l'expression du mari - d'où, là encore, la relance faite au mari, avant toute traduction à la psychiatre ${ }^{6}$. Elle sait en effet que celui-ci peine à s'exprimer et combien, a contrario, sa femme est prompte à " parler toujours à sa place".

L'on peut certes considérer, dans une perspective critique, que l'interprète se trompe ici partiellement - il est en effet possible de signaler dans un certificat médical qu'un demandeur d'asile n'est pas en capacité de répondre de manière cohérente aux questions qui lui sont posées. L'on peut aussi faire valoir que, en intervenant comme elle le fait, l'interprète se mêle de questions qui ne la regardent pas tout à fait.

Mais, d'une part, ce serait ne pas voir que c'est un risque qu'elle connaît fort bien et qu'elle cherche à éviter. Lorsqu'elle décrypte l'embarras suscité par la demande de la femme, elle me dit que si elle avait pu, elle l'aurait reprise pour lui signifier le caractère impropre de sa demande. Et si elle s'est retenue, c'est précisément pour rester à sa place d'interprète : "Je peux pas lui dire "Mais Madame...,", moi je suis juste [là] pour traduire. " D'autre part, ce serait ne pas voir que du positionnement de l'interprète dépend en effet la capacité des uns et des autres à parler ou pas.

Ce que j'aimerais faire valoir ici, c'est que ces critiques ou ces limites qui peuvent être opposées à l'interprète le sont depuis une perspective exogène, qui risque toujours de ne pas voir que son travail n'engage pas seulement les aspects sémiotiques d'une interaction, mais également ses dimensions épistémiques, relationnelles, morales, organisationnelles, etc. L'interprète doit, comme tous les autres protagonistes de cette consultation, composer avec ces entités hétérogènes et cette hétérogénéité est bien ce qui risque de les faire trébucher à chaque pas. 


\section{Percevoir des affordances langagières}

La psychiatre clôt ce premier moment de la consultation consacré à l'avancement des démarches administratives en résumant ce qu'elle a mis dans ce certificat. Elle enchaîne ensuite sur les questions de santé. Avant de m'y engager à sa suite, j'aimerais revenir sur la manière dont l'interprète négocie une difficulté de traduction : le terme "gravité " utilisé par la psychiatre lui manque.

La psychiatre : "Voilà, où j'ai insisté, je résume simplement sur la gravité de l'état de santé de votre mari. "

L'interprète : "J'ai encore mis... Comment on dit en serbe? ... Que c'est mal pour lui ce qui s'est passé, ce que vous avez dit, qu'il a des absences [izgubi]. Voilà."

L'interprète hésite, s'interroge à haute voix et partage ainsi son trouble avec le couple, comme si elle sollicitait son aide. Si cette demande d'aide peut sembler incongrue, la femme et son mari ne parlant pas français, force est de constater que c'est pourtant bien grâce à eux que l'interprète va trouver une solution à son problème.

Après avoir tenté une première traduction très approximative (" c'est mal pour lui ce qui s'est passé ") qui ne la satisfait manifestement pas, elle recourt à un déictique ("ce que vous avez dit»), pointant ainsi le fait que "gravité " vaut pour un terme employé par le couple. II ne lui reste ensuite plus qu'à expliciter la référence : "Ce que vous avez dit, [c'est qu'il a des] absences [izgubi]».

Izgubi n'est certes pas la traduction du terme français "gravité ". II présente en revanche l'immense intérêt d'être déjà disponible en serbe, alors que "gravité " ne l'est pas (encore). II a, en effet, été utilisé deux minutes plus tôt par le mari qui renchérissait à la demande faite par sa femme à la psychiatre, signalant qu'il avait des « absences [izgubi] » (cf. supra).

L'effet produit (identité des propos du couple et de la psychiatre) est certainement on ne peut plus rassurant pour le premier : "voilà que ce que vous avez dit " se trouve précisément dans le certificat qu'elle a rédigé.

En rendant visible cette manière de faire, il ne s'agit pas pour moi de dévoiler et de dénoncer ce qui ne serait qu'un artefact ou une supercherie, que "gravité " 
n'est pas exactement izgubi. Cela, l'interprète ne le sait que trop bien. J'entends plutôt montrer comment, pour une part, traduire c'est percevoir des " affordances " langagières. Cette notion forgée par le psychologue américain James Gibson (2014/1979) désigne les possibilités d'action offertes par un environnement. Elles ne sont si seulement subjectives ou mentales (dans la tête de celui qui les perçoit) ni seulement objectives, physiques ou matérielles (dans les objets et les choses de cet environnement). Pour reprendre l'un des exemples de James Gibson, la boîte aux lettres " invite [afford] un humain qui écrit des lettres à envoyer des lettres au sein d'une communauté dotée d'un système postal ". Mais l'affordance de la boîte aux lettres " ne change pas en fonction des changements de besoin de l'observateur »(Gibson, 2014/1979). Ce n'est pas parce que je n'ai pas de lettre à poster que la boîte aux lettres au coin de ma rue ne continue pas à m'inviter à poster des lettres. En revanche, faute de lettre à poster, je peux ne pas percevoir cette affordance, ne pas lui être attentive.

En proposant de parler d'affordances langagières, j'appréhende la langue en tant qu'elle est un "environnement" avec lequel l'interprète et les autres protagonistes sont " en transaction " (Dewey, 1993/1938). Certains éléments sont saisis comme autant de prises pour s'orienter, à l'instar du terme izgubi. D'autres ne sont pas perçus et risquent alors de passer par pertes et profits. C'est le cas lorsque l'interprète, tout comme la psychiatre à sa suite, considèrent que la mention par le mari de ce qu'il a des " absences" ouvre sur un autre sujet.

Cette approche écologique présente l'intérêt de donner à voir que, pour autant que les affordances sont objectives et existent bel et bien, elles peuvent ne pas être perçues. Tout engagement dans le monde laisse irrémédiablement derrière lui un reste.

\section{Hantises. Des restes qui résistent}

La psychiatre en vient donc à aborder les questions de santé. Elle commence en disant qu'elle a une "question très précise " à poser, sans doute pour requérir toute l'attention et la précision de l'interprète, puis dit : "Quand on s'est vus la dernière fois, je vous ai proposé d'essayer un médicament qui pouvait éventuellement aider un peu pour les cauchemars. Donc je voulais savoir si y avait une différence, même minime, ou pas du tout de différence. Comment vous supportez ce médicament puisqu'il y a des gouttes et un comprimé?»

L'interprète : "La dernière fois elle vous a donné des gouttes. " 
Le mari hoche la tête, la femme dit: "Oui. »

L'interprète : "Et un médicament à prendre, est-ce qu'il y a, elle dit, une différence : un peu, plus ou rien?"

Le mari : "Non, une petite différence, il n'y en a pas vraiment. "

L'interprète : " Juste un peu?»

Le mari : "Un peu, y a pas beaucoup parce que moi j'ai ces cauchemars et je dors peu."

L'interprète : "Et pour les cauchemars, elle l'a donné. »

Le mari : "Oui, c'est ce que je dis. »

L'interprète : "Ça n'a pas diminué ça?»

Le mari : " Non, ça a un peu diminué, mais c'est pas encore ça. »

L'interprète : "C'est ce qu'elle a dit un peu avant, elle dit qu'elle a mis que votre état s'aggrave [inaudible]. »

L'homme acquiesce. Sa femme intervient: " J'ai remarqué cela moi aussi."

L'interprète : "Et ça, elle l'a mis sur ce papier qui est parti à Paris. "

L'interprète démarre ensuite sa traduction pour la psychiatre.

II| conviendrait alors de montrer que ce n'est pas parce que l'interprète et la psychiatre partagent ce même impératif qu'elles se rencontrent nécessairement. Le partage est ce qui réunit et sépare tout à la fois.
Dans le cadre imparti ici, je ne peux m'arrêter sur la manière dont, au cours de notre entretien, l'interprète fait valoir combien ses traductions et les relances faites au mari visent à répondre à cet impératif de précision qu'elle partage avec la psychiatre?

Je relèverai seulement un aspect de cette séquence : le fait que sinon le terme "gravité ", à tout le moins le verbe "s'aggraver" revient à l'interprète. Celle-ci s'autorise alors à faire une incise et à revenir en arrière, en signalant cette rupture 
temporelle à l'aide de marqueurs ad hoc : recours au passé (" elle a dit "), antériorité affichée du propos de la psychiatre (" un peu avant"). Ce sur quoi l'interprète a achoppé ne disparaît pas vraiment : ce sont autant de restes qui la hantent et qui sont susceptibles de revenir sur le devant de la scène, par exemple, lorsqu'une meilleure solution de traduction s'offre à elle. Surtout, il convient de remarquer que la traduction du terme sur lequel elle avait jusque-là achoppé charrie à sa suite le contexte dans lequel il avait été initialement énoncé. Ce faisant, elle risque aussi bien de contribuer à rendre l'organisation de la consultation moins lisible. Alors que la psychiatre en a fini avec les démarches administratives, voilà que l'interprète y revient. Si le retour de ces restes permet à l'interprète de ménager des continuités, il est toujours aussi susceptible de générer de nouvelles discontinuités. La solution trouvée par l'interprète à ce moment-là de la consultation en est bien une, mais elle risque de créer un autre problème.

L'approche écologique que je déploie ici invite à se départir d'une conception finaliste du travail de l'interprète. Ce n'est pas parce que, en bout de course, il s'avère que pas grandchose n'est perdu par l'interprète, qu'il est possible de passer outre les innombrables discontinuités et décrochages qui maillent ces interactions. Les développements qui précèdent montrent combien ils sont constitutifs du travail de l'interprète. Dès lors, il n'y a plus lieu de s'effrayer des écarts et des pertes inhérents à toute traduction. En revanche, il convient de penser le caractère risqué des interventions de l'interprète. L'interprète se tient toujours dans un équilibre précaire. Les prises dont elle se saisit en situation peuvent se dérober et les solutions qu'elle entrevoit s'avérer ne pas en être tout à fait; toute perspective sur la situation laissant nécessairement derrière elle un reste. D

\section{BBLLOGRAPHE}

Cahour, B. et Licoppe, C. (2010). Confrontations aux traces de son activité. Revue d'anthropologie des connaissances, 4(2), 243-253.

Cassin, B. (2016). Éloge de la traduction. Compliquer I'universel. Paris : Fayard.
Dewey, J. (1993/1938).

Logique. La théorie de l'enquête. Paris : PUF. Gibson, J. J. (2014/1979). Approche écologique de la perception visuelle. Paris : Dehors.

Haraway, D. (2016). Staying with the trouble. Making Kin in the Chthulucene. Durham and London: Duke University Press.
Hennion, A. (2015). La médiation : un métier, un slogan ou bien une autre définition de la politique? Informations sociales, 4(190), 116-123.

Pury Toumi (de), S. (2005). Comment on dit dans ta langue? Pratiques ethnopsychiatriques. Paris : Les Empêcheurs de penser en rond. 